\title{
Approche pédologique du milieu prairial en Margeride
}

\author{
M. Bornand et D. Menier \\ Laboratoire INRA-ENSA, Science du Sol, 34060 Montpellier Cedex, France
}

(reçu le 24-6-1986, accepté le 28-9-1988)

Résumé - Pour la bordure du sud du Massif central français, une approche cartographique à l'échelle du $1 / 250000 \mathrm{e}$ permis d'affiner nos données sur la distribution séquentielle des sols, en relation avec la végétation, le climat et l'altitude. Un découpage en grandes régions naturelles, bien caractérisées sur le plan lithologique, géomorphologique et climatique a pu être réalisé. Ainsi, il a été possible de définir les lois d'organisation et de répartition des sols. Cette approche à petite échelle a été complétée par une approche détaillée, mais discontinue, à l'échelle d'aires de références représentatives de ces milieux qui a permis de préciser la typologie des sols et les principales caractéristiques du milieu qui peuvent jouer le rôle de facteurs limitants : texture, profondeur, degré d'hydromorphie, sensibilité relative à la sécheresse, pentes et situation topographique.

L'approche méthodologique proposée permet de réunir de bonnes conditions d'extrapolation pour passer d'une caractérisation régionale à base génétique à une caractérisation agronomique des sols qui pourra rendre compte de leur comportement possible vis-à-vis d'une culture donnée, ici, la prairie.

cartographie des sols - typologie agronomique - prairies (France) - montagnes cristallines

Summary - Pedological approach to grass land in southern french Massif central (Margeride). Regional mapping at a scale of 1/250 000 provided more detailed unformation on sequential soil distribution in relation to vegetation, altitude and climate. Regions were divided up according to specific lithological, geomorphological and climatic characteristics, thus permitting a definition to be made of factors determining soil organisation and distribution.

This small-scale approach was complemented by a detailed but discontinuous study of sample areas within these natural regions. The latter approach enabled soil typology to be determined, and also the main characteristics of the area which might act as limiting factors : soil texture, depth, degree of hydromorphy, relative sensitivity to drought, slope and local topography.

The methodological approach presented here enables an extrapolation to be made from a regional characterisation of soil on a genetic basis to an agronomic characterisation of soil including its possible reaction to a specific culture, in this case grass land.

soil mapping - agronomic typology - grassland (France) - crystalline mountains

\section{Introduction}

Située au coeur du Massif central, la Margeride couvre environ 170000 ha et s'étend à l'ouest des plateaux du Velay et de l'Ardèche, au nord des plateaux calcaires des grands Causses et à l'Est des plateaux volcaniques d'Aubrac. Elle s'étend essentiellement dans le département de la Lozère mais déborde quelque peu sur ceux du Cantal et de la Haute-Loire (Fig. 1). C'est une région de moyenne montagne ( 1000 à $1550 \mathrm{~m}$ ), essentiel- lement granitique, à climat intra montagnard et à vocation d'élevage (de Montard et al., 1983) : surface agricole utile (SAU) et surface toujours en herbe (STH) occupent respectivement $87 \%$ et $74 \%$ de la superficie totale (RGA, 1979).

Le relief de Margeride, monotone et peu vigoureux, n'est qu'une succession de fluves et d'interfluves de faibles dimensions et s'avère globalement propice à la mécanisation de l'exploitation des prairies. Cependant, le facteur "sol» apparaît défavorable à la production herbagère. Toujours 


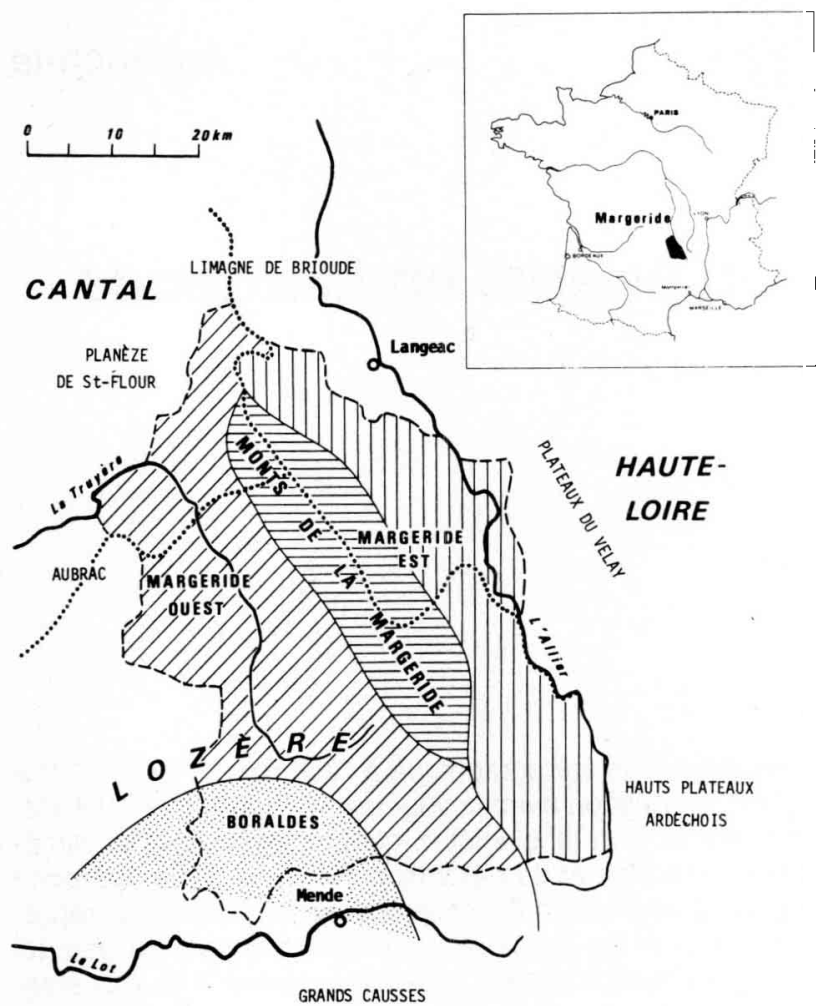

Fig. 1. Localisation de la Margeride en France. Les principales régions naturelles.

acides, les sols sont souvent hydromorphes daris les fonds de vallées. Sensibles à la sécheresse estivale sur les versants, ils disposent d'une réserve utile en eau limitée : profondeur faible, texture grossière, pierrosité importante. Le climat constitue aussi un facteur limitant pour la productivité des prairies, à cause de la brièveté de la saison végétative et surtout à cause de la fréquence des gelées printanières retardant la récolte de 1 re coupe, mais aussi du fait de la sécheresse estivale entraînant des regains médiocres (INRA, 1983).

L'objectif de cet article est de présenter une méthode d'approche pédologique du milieu prairial. Cette approche débouche sur une typologie des sols de Margeride (Menier, 1985), qui constituera pour l'agronome un outil de base pour la caractérisation des comportements prairiaux. Ces comportements ne seront pas étudiés dans le présent article et feront l'objet d'un développement ultérieur.

\section{Méthode d'étude du milieu herbager}

Les facteurs intervenant sur la production herbagère sont à la fois complexes et interdépendants. Notre approche met en œuvre une méhode qui aide à mieux déterminer le rôle de ces différents facteurs sur cette production, Jusqu'à présent, les nombreux travaux réalisés dans ce domaine ont quelque peu négligé l'intégration du facteur «sol». La méthode adoptée essaye de prendre en compte aussi bien le facteur "sol» que les autres facteurs du milieu pouvant avoir une influence sur la production herbagère.

La méthode est basée sur un ensemble d'investigations cartographiques destinées à prende en compte les facteurs du milieu physique. Elle comporte 3 phases successives qui se situent à deux niveaux d'échelles complémentaires et qui sont résumées ci-après.

\section{jre phase}

II s'agit de la définition et de la délimitation des unités naturelles régionales que l'on peut considérer comme homogènes vis-à-vis des facteurs du milieu physique. Cette étape s'appuie d'abord sur la consultation des documents classiques déjà existants (carte géologique, carte lithomorphologique, carte de la végétation, documents bioclimatiques) et sur leur confrontation avec le terrain. II est bien évident que les aspects pédologiques ont eu une place importante dans ce zonage. Pour le secteur concerné, en l'absence de document existant sur les sols, une étude de reconnaissance des sols au $1 / 250000^{\circ}$ (Bonfils et Legros, 1985) a du être entreprise afin de réunir les éléments indispensables à la réalisation d'un zonage du milieu régional intégrant ce facteur sol.

\section{$2^{e}$ phase}

C'est le choix et la sélection des secteurs représentatifs des régions naturelles : dans chacune des régions naturelles précédemment délimitées, un certain nombre de secteurs sont choisis comme références d'études. Les superficies concernées (100 à $200 \mathrm{ha}$ ) correspondent le plus souvent aux surfaces que peuvent occuper une ou plusieurs exploitations agricoles. Le choix des exploitations agricoles de référence tient compte :

- de la représentativité de l'exploitation agricole par rapport à l'ensemble de la région naturelle;

- de la diversité des roches mères présentes (granite porphyroïde, leucogranite, gneiss-micaschiste), des tranches d'altitudes (comprises entre 950 et $1300 \mathrm{~m}$ ) et enfin des situations géomorphologiques de Margeride (types de vallées et modèles des versants.

\section{3e phase}

Elle correspond à l'étude détaillée des sols de chaque secteur de référence avec une mise au point d'une typologie simplifiée des sols : cette étude, effectuée à une échelle voisine du $1 / 10000^{\circ}$ permet de comprendre l'organisation et la répartition spatiale des sols pour les paysages et les régions à caractériser; elle conduit à la définition de toposéquences et à la construction de blocs diagrammes modélisant la distribution des sols au sein des unités géomorphologiques les plus typiques. Elle aboutit à la réalisation d'une carte des sols permettant, pour chacune des situations inventoriées, de défi- 
nir et de localiser les diverses catégories de sols représentées.

La distinction entre ces catégories de sols est basée sur des critères faciles à mettre en œuvre par les agronomes et par les non-pédologues. La caractérisation physique et chimique simple auquelle elle conduit correspond à une typologie simplifiée des sols, qui voudrait constituer un outil permettant de rendre compte au mieux du comportement des prairies en Margeride. Ce comportement sera d'autant mieux approché que les critères de caractérisation choisis ont une signification biologique réelle. C'est pourquoi les critères retenus prennent largement en compte différents aspects comme la disponibilité en eau pour la plante, ainsi que la pente ou I'hydromorphie (facteurs souvent limitants pour la production herbagère ou fortement contraignants pour les possibilités d'accès à la parcelle dans le cas des prairies de Margeride).

\section{$4^{e}$ phase}

C'est la phase agronomique de la démarche et elle ne sera pas exposée : la typologie des sols élaborée lors des 3 phase précédentes sert de canevas de base à l'évaluation des comportements prairiaux qui peut être réalisée selon le principe de l'enquête agronomique prenant en compte notamment la qualité floristique et les rendements herbagers.

\section{Résultats}

La méthodologie que nous venons d'expliciter schématiquement a permis de distinguer des régions naturelles bien caractéristiques, au sein desquelles l'on a pu procéder à une typologie des sols qui est présentée ci-après.

\section{Les quatre régions naturelles de la Margeride}

\section{Description et caractéristiques principales}

L'ensemble des facteurs physiques du milieu permet de mettre en évidence de façon nette 4 régions naturelles sur notre domaine d'étude (Fig.1).

Par tous les facteurs étudiés, la région des Boraldes métamorphiques au sud-ouest se démarque de la Margeride granitique au nord. Son relief, fortement vallonné, favorisant le drainage naturel des eaux, explique la fréquence des sols de pente et des affleurements défavorables à la mécanisation de l'exploitation des prairies, et l'absence relative de sols hydromorphes. De plus, son altitude moindre $(<1100 \mathrm{~m})$ entraîne une saison végétative plus longue ( 7 mois).

La Margeride, stricto sensu, forme un ensemble très homogène. Seuls les monts de la Margeride, d'altitude plus élevée (>1200 m) constituent une dorsale d'orientation nord-ouest/sud-est et se distinguent des plateaux granitiques à l'est et à l'ouest, d'altitude inférieure à $1200 \mathrm{~m}$ (Fel, 1962; 1983). Le climat, plus rude, est caractérisé par une saison végétative plus courte (4 à 5 mois) mais un déficit hydrique estival moins marqué. II explique l'extension des sols humifères : le climat froid entraîne une activité biologique faible et une minéralisation lente de la matière organique, d'autant plus que la végétation est acidifiante (lande à callune).

La Margeride-Est et la Margeride-Ouest constituent la zone où dominent largement les sols bruns acides et les sols bruns ocreux. Entre ces 2 régions, la distinction est difficile à opérer. Seules les caractéristiques climatologiques sur 30 ans indiquent un déficit hydrique estival un peu plus marqué pour la Margeride-Est protégée des influences océaniques par les monts de la Margeride (Estienne, 1956; Gachon, 1946).

Types de paysages et critères dominants d'évolution des sols

Les régions ainsi définies présentent une géomorphologie et des modelés bien caractéristiques avec des paysages au sein desquels l'on observe une organisation et un ordonnancement typique des cultures et des forêts en liaison avec les caractères propres des sols.

Dans les Boraldes métamorphiques (Fig. 2), la morphologie est beaucoup plus accusée qu'en Margeride (s.s.). Typiquement, l'on note de longs versants en pente faible qui sont délimités par des pentes très fortes, déterminant des vallées très encaissées, où l'opposition «adret-ubac» est nette. La forêt occupe tout l'ubac, alors qu'elle se limite aux zones de pentes fortes à l'adret. Cultures et prairies sont le plus souvent situées audessus des pentes fortes occupées par la forêt.

L'altitude reste inférieure à 1 000-1 $100 \mathrm{~m}$, de sorte que la rigueur climatique ne provoque pas de blocage prolongé de la minéralisation de la matière organique et de phénomènes marqués d'accumulation, même sur les sommets des versants. Au contraire, ce sont plutôt les phénomènes d'érosion et de rajeunissement qui jouent les rôles dominants dans la répartition et l'évolution des sols sur les pentes les plus fortes.

En Margeride granitique (Fig. 3) les altitudes moyennes sont plus élevées (1 100-1300 m), les différences de relief sont beaucoup moins accusées et la morphologie d'ensemble est assez molle. Les vallées largement ouvertes sont peu 


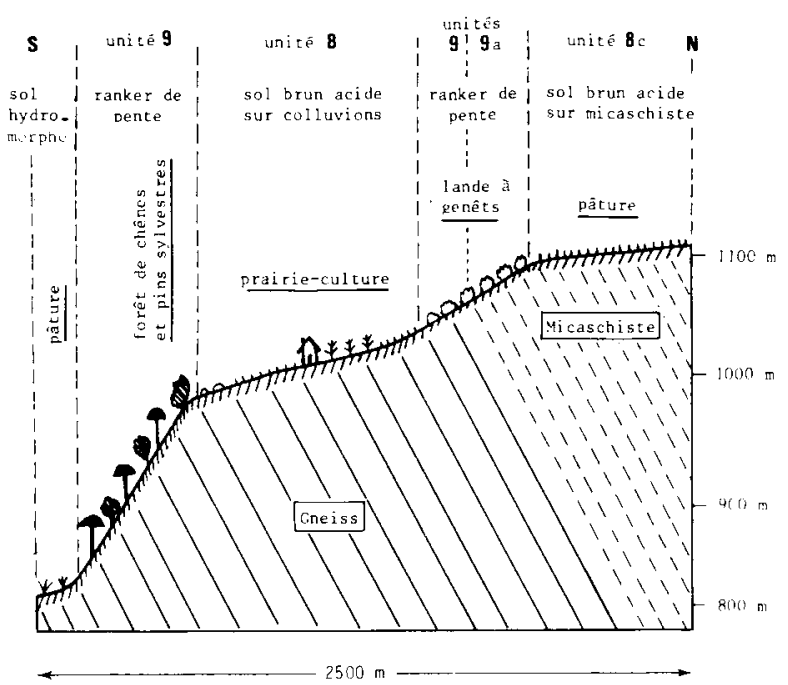

Fig. 2. Toposéquence simplifiée dans les Boraldes métamorphiques.

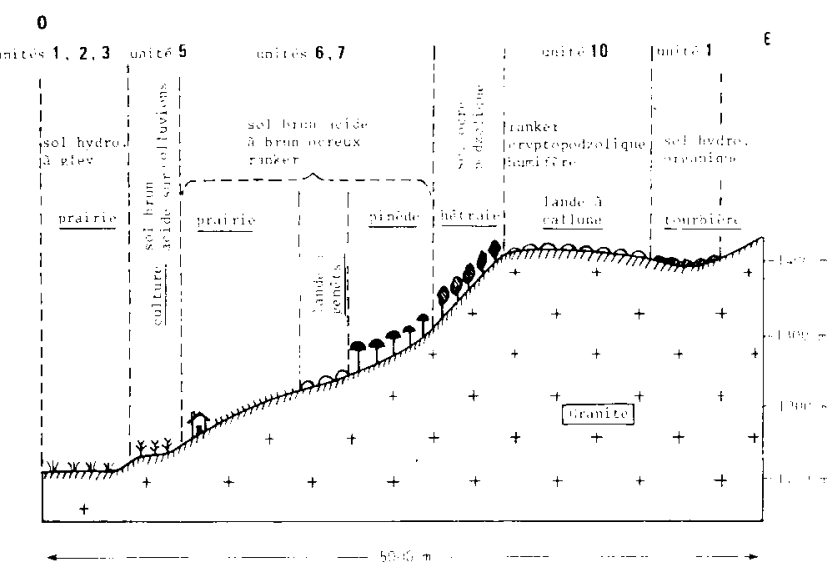

Fig. 3. Toposéquence simplifiée en Margeride granitique.

profondes et faiblement recreusées; elles sont encadrées par d'assez longs versants aux pentes faibles, où l'effet adret-ubac est peu sensible. Dans ces conditions, l'allure des paysages est bien différente de celle des Boraldes : les secteurs en pentes les plus fortes sont situés loin des vallées, plutôt vers le sommet des versants; ils sont occupés par les forêts et par les landes. Au contraire, les cultures et prairies s'étendent sur les bas de versants en-dessous de ces secteurs sommitaux, domaine de la végétation naturelle.

Dans ces situations, rudesse du climat, type de roche et morphologie molle se conjuguent pour favoriser le développement et l'extension de sols plus profonds, où d'importants phénomènes d'accumulation de matière organique peuvent se produire (dans les bas fonds et en zone de plateaux d'altitude).
L'ensemble de ces caractéristiques et de ces relations est présenté de manière synthétique dans le Tableau I.

\section{Typologie des sols de Margeride}

A l'intérieur des unités lithomorphologiques de paysages précédemment définies, un certain nombre de critères simples ont été choisis à partir desquels il est possible de distinguer, de définir et de caractériser les principales unités de sols existant en Margeride.

\section{Principaux critères de caractérisation des unités}

Les critères retenus peuvent se regrouper en 2 ensembles : critères de situation et critères liés aux sols, qui sont regroupés dans le Tableau II.

Critères de situation. La géomorphologie représente un des critères majeurs de distinction. C'est ainsi que l'on a été amené à différencier les unités occupant une position en fonds de vallées ou en talus de raccordements avec les versants de celles situées sur les versants S.S. ou sur les tertres (secteurs représentants des micro-bombements de 1 à $3 \mathrm{~m}$ ou petites élévations de terrains, sans doute d'origine à la fois colluviale et anthropique, situées en position de transition entre les bas de versants et la vallée proprement dite).

La situation altitudinale correspond, par ailleurs, à un autre critère important. L'augmentation d'altitude et la rigueur du climat dans la Margeride entraînent en effet un défaut de minéralisation s'accompagnant d'une accumulation de matière organique entraînant l'expression de processus d'évolution des sols de plus en plus marqués dans les sols de versants d'altitude (processus de podzolisation liés à la présence de substances organiques, actions entraînant la possibilité de dégradation des argiles et l'apparition d'aluminium libre).

Critères propres aux sols. Pour les fonds de vallées, c'est l'intensité des manifestations des phénomènes d'engorgement qui est déterminante pour la distinction entre unités. Ainsi, on a tenu compte de la présence ou de l'absence d'horizons tourbeux $(\mathrm{H})$ ainsi que de la profondeur d'apparition des phénomènes de réduction et d'oxydoréduction du fer dans le profil (présence d'horizon à gley $\mathrm{G}$ ).

Pour les versants, la nature de la roche mère joue un rôle très important. Elle intervient moins par sa géochimie que par sa susceptibilité à l'altération, qui commande plus ou moins directement la profondeur des sols. Ainsi, on a été amené à 
Tableau I. Caractérisation des régions naturelles en Margeride. Base pour le choix des exploitations de référence.

\begin{tabular}{|c|c|c|c|c|c|c|c|}
\hline RÉGIONS MATURELLES & GEEOLOGIE & TOPOGRAPHIE & CLIMAT & PHYTOCLIMAX & Pëdoc l imax & Climax stationnel & $\begin{array}{l}\text { EXPLOITATIONS AGRICOLES : } \\
\text { SOUS-SECTEURS DE REFERENCE }\end{array}$ \\
\hline $\begin{array}{l}\text { Monts de la } \\
\text {.. Margeride }\end{array}$ & Granite & $\begin{array}{l}\text { Vallees peu profon- } \\
\text { des } \\
\text { Pentes faivles }\end{array}$ & $\begin{array}{l}\text {-Alt. } 1500 \text { à } 1200 \mathrm{~m} \\
\text { - Sa ison végétative } \\
\text { très breve : } 4 \text { à } 5 \\
\text { mo is } \\
\text {-Secheresse estivale } \\
\text { atténuée }\end{array}$ & $\begin{array}{l}\text {-Pelouse pseudo- } \\
\text { alpine }(1400 \mathrm{~m}) \\
\text {-Hêtraie }(1400 \mathrm{~m})\end{array}$ & $\begin{array}{l}\text { - Ranker cryptopodzo- } \\
\text { lique: sol assez } \\
\text { profond, humifére. } \\
\text { Sol ocre-podzolique } \\
\text { sous hêtraze }\end{array}$ & - Tourbières fréquentes & 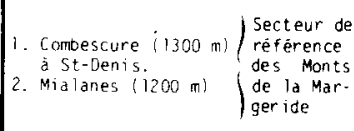 \\
\hline Marger ide Est & Granite & $\begin{array}{l}\text { Vallées peu profon- } \\
\text { des } \\
\text { Pentes faibles a } \\
\text { moyennes }\end{array}$ & $\begin{array}{l}\text {-Alt. } 1200 \text { à } 900 \mathrm{~m} \\
\text { - Saison vêgétative } \\
\text { brève: } 5 \text { à } 6 \text { mois } \\
\text {-Sécheresse estivale } \\
\text { trés marquée }\end{array}$ & Hêtrale & $\begin{array}{l}\text {-Sol brun acide à } \\
\text { brun ocreux : } \\
\text { sol peu profond, peu } \\
\text { humifere a texture } \\
\text { trés grosstere }\end{array}$ & $\begin{array}{l}\text {-Sols hydromorphes en } \\
\text { fond de talweg. } \\
\text { - Sols de pentes et af- } \\
\text { fleurements peu nom-. } \\
\text { breux }\end{array}$ & 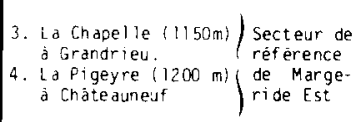 \\
\hline Marger ide Ouest & Granite & $\begin{array}{l}\text { Vallees peu profon- } \\
\text { des } \\
\text { Pentes falbles à } \\
\text { moyennes }\end{array}$ & $\begin{array}{l}\text {-Alt. } 1200 \text { à } 1000 \text { m } \\
\text { - Saison végetative } \\
\text { breve: } 5 \text { à } 6 \text { mois } \\
\text {-Secheresse estivale } \\
\text { marquee }\end{array}$ & Hétraie & $\begin{array}{l}\text {-5ol brun acide à } \\
\text { brun ocreux : } \\
\text { sol peu profond peu } \\
\text { humifere, à texture } \\
\text { trés grossiere }\end{array}$ & $\begin{array}{l}\text { - Sols hydromorphes en } \\
\text { fond de talweg. } \\
\text {-Sols de pentes et af- } \\
\text { fleurements peu nom- } \\
\text { breux }\end{array}$ & 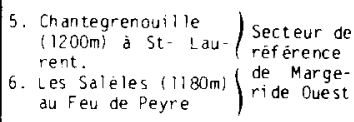 \\
\hline $\begin{array}{l}\text { Boraldes } \\
\text { Sud Aubrac }\end{array}$ & $\begin{array}{l}\text { Gneiss } \\
\text { Micaschistes }\end{array}$ & $\begin{array}{l}\text { Vallees encaissées } \\
\text { Pentes fortes }\end{array}$ & $\begin{array}{l}\text {-Alt. } 1100 \text { à } 800 \mathrm{~m} \\
\text {-Saison vegetative } \\
\text { moyenne : } 7 \text { mois } \\
\text {-Sécheresse estivale } \\
\text { marquée }\end{array}$ & chênaie & $\begin{array}{l}\text {-sol brun acide: } \\
\text { sol peu profond, } \\
\text { peu humifêre, à } \\
\text { texture grossière }\end{array}$ & $\begin{array}{l}\text {-Peu de sols hydromor- } \\
\text { phes. } \\
\text { - Nombreux sols de pen- } \\
\text { tes et affleurements }\end{array}$ & $\begin{array}{l}\text { 7. Le Massibert }(980 \mathrm{~m}) \text { Secteur de } \\
\text { réference } \\
\text { a Chirac. } \\
\text { 8. Fabreges }(950 \mathrm{~m}) \text { a } \\
\text { Chirac }\end{array}$ \\
\hline
\end{tabular}

Tableau II. Critères de caractérisation des unités de sol et de paysage.

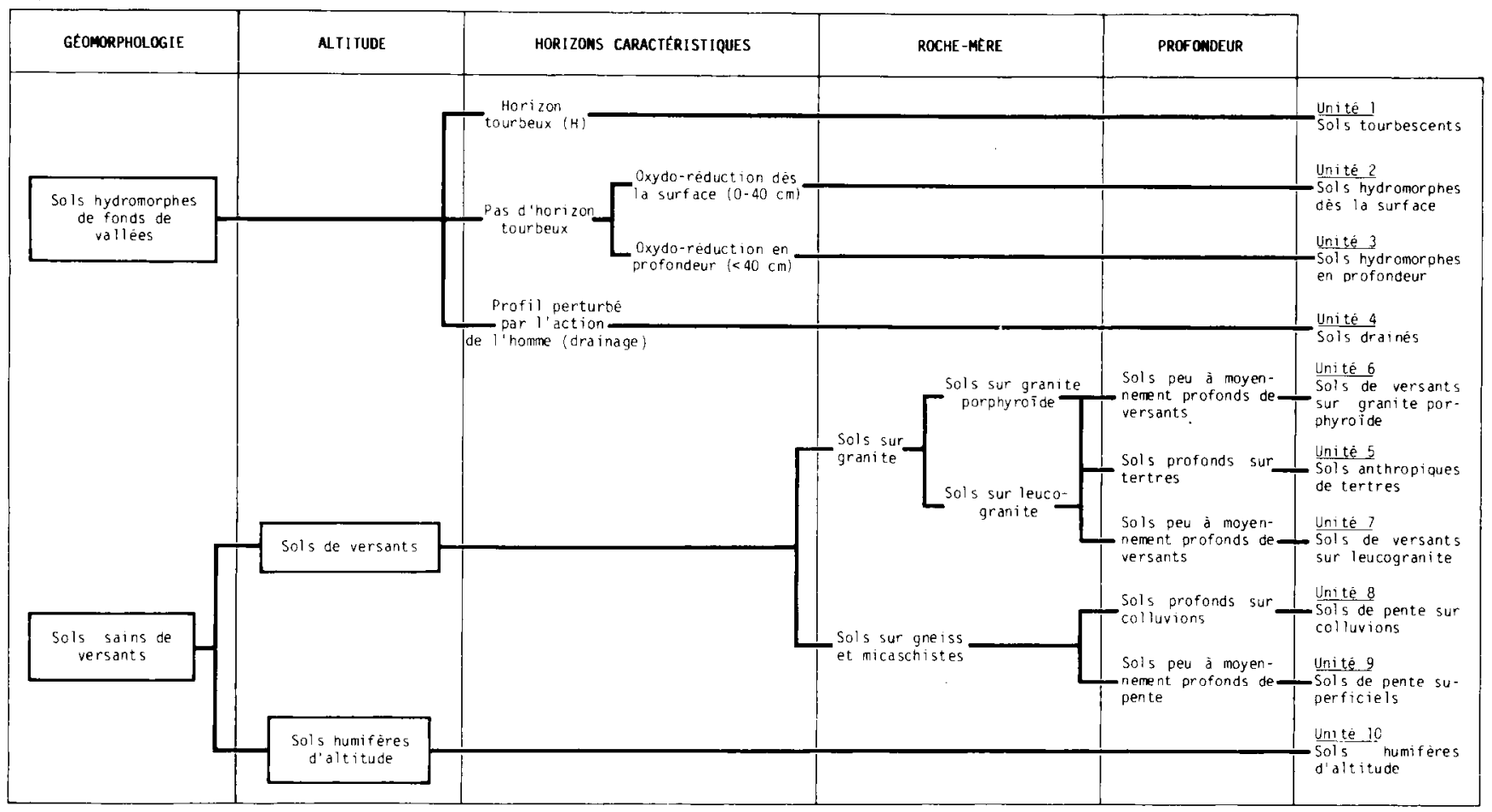

distinguer les sols de versants sur gneiss-micaschiste, sur granite porphyroïde et sur leucogranite.

D'autres critères comme ceux de texture, de pierrosité, de type d'arène ainsi que les caractéristiques chimiques sont aussi intervenus secon-dairement pour la caractérisation complémentaire des diverses unités de sols.

De plus, un autre critère a été considéré comme essentiel, pour la distinction et la caractéri- sation des unités de sols, il est représenté par la végétation naturelle. L'observation de la flore a permis de déterminer quelques espèces indicatrices liées aux principales unités de sols. II n'existe que peu d'espèces caractéristiques (espèces présentes exclusivement sur un type de sol), mais beaucoup d'espèces préférantes (espèces dont la présence permet d'identifier un type de sol avec une probabilité supérieure à 0,8 ). Ces espèces constituent de bons indicateurs de la disponibilité 
en eau du sol : elles caractérisent notamment le degré d'engorgement des sols de fond de vallées et aussi l'épaisseur plus ou moins grande des sols de versants.

Ce critère est détaillé dans l'ensemble des Tableaux IIla et IIlb, sous la rubrique «Espèces préférantes» associées à chacun des principales unités de sols.

Les unités de sols : description et caractéristiques. Nous passerons successivement en revue les 2 grands ensembles de sols qui ont été distingués : sols des fonds de vallées, puis sols des versants.

\section{Les sols de fonds de vallées}

Les diverses unités de sols des fonds de vallées se distinguent par la présence (unité 1) ou l'absence de niveau tourbeux, ainsi que par la profondeur d'apparition des phénomènes d'hydromorphie, soit dès la surface (unité 2), soit en profondeur (unité 3). L'unité 4 regroupe l'ensemble des sols drainés, très hétérogènes du point de vue du développement des profils, mais qui sont assez bien typés par un comportement caractéristique des prairies.
Le Tableau Illa présente de manière synthétique l'ensemble des caractéristiques de chacune de ces unités de sols, en insistant sur celles que l'on considère comme les plus importantes pour le diagnostic de la typologie : position précise occupée dans le modelé local et espèces préférentes associées; caractéristiques morphologiques des profils avec horizons majeurs observés; caractéristiques des fonctionnements hydriques; contraintes majeures qui découlent de ces propriétés pour la mise en place ou l'exploitation agronomique des prairies au sein des diverses unités définies.

\section{Géomorphologie. Origine des matériaux}

Tous ces sols occupent des vallées peu larges (50 à $500 \mathrm{~m}$ ) dont les pentes transversales et longitudinales sont faibles à nulles; ce qui influe directement sur les possibilités d'écoulement et d'évacuation des eaux en excès. Le substrat est toujours allochtone, d'origine alluvio-colluviale, il est constitué par une arène granitique, partiellement altérée et transformée en une argile compacte et peu perméable (Sattler, 1984); d'où l'existence dans ces sols d'horizons profonds, asphyxiants,

Tableau Illa. Typologie simplifiée des sols de vallées en Margeride.

\begin{tabular}{|c|c|c|c|c|}
\hline $\begin{array}{l}\text { CRITERES DE UNITES DE SOL } \\
\text { CARACTERISATION }\end{array}$ & $\begin{array}{c}\text { UN! TE l } \\
\text { SOls tourbescents } \\
\text { (SOLS HYDROMORPHES ORGANIQUES) }\end{array}$ & $\begin{array}{l}\text { UN: TÉ } 2 \\
\text { Sols hydromorphes dés la surf ace } \\
\text { (SOLS HYDROMORPHES A GLEY) }\end{array}$ & $\begin{array}{l}\text { UNITE } \\
\text { Sols hydromorphes en profondeur } \\
\text { (SOLS HYDROMORPHES A GLEY) }\end{array}$ & $\begin{array}{l}\text { UNIIE } 4 \\
\text { Sols draines }\end{array}$ \\
\hline $\begin{array}{l}\text { CRITERES DE SURFACES } \\
\text {. Géomorphologie } \\
\text {. Occupation du sol: } \\
\text {. Espèces préferentes : }\end{array}$ & 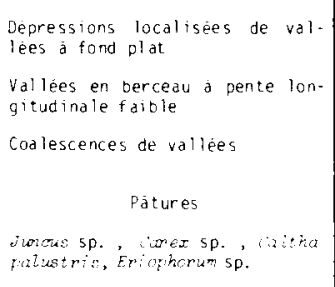 & 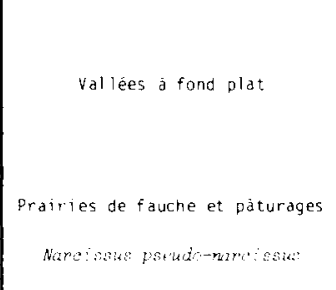 & 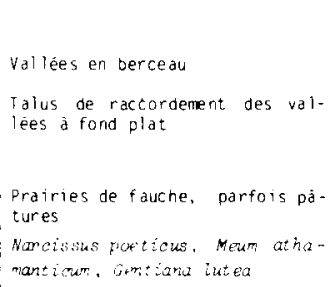 & $\begin{array}{l}\text { Voir UNITES } 1,2 \text { ou } 3 \\
\text { Prairies de fauche } \\
\text { Evolution vers flore } \\
\text { de l'UNItE } 3\end{array}$ \\
\hline $\begin{array}{l}\text { CARACIERIS":OUES } \\
\text { MORPHOLOGIOUES }\end{array}$ & 110 Cm & 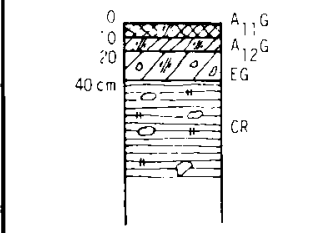 & OLG & Pas de profil type \\
\hline $\begin{array}{l}\text { CARAC 'ERTST:OUES HYORIQUES } \\
\text { - Type d'engorgement } \\
\text { - Durée de submersion } \\
\text { Réserve utile }\end{array}$ & $\begin{array}{l}\text { Géneralise, quasi permanent dès } \\
\text { la surface } \\
\text { Submersion prolongee } \geqslant 6 \text { mois }\end{array}$ & $\begin{array}{l}\text { Géneraliso, temporaire en surfa- } \\
\text { ce et permanent au-dessous de } \\
40 \mathrm{~cm} \\
\text { Submersion prolongée : } 3 \text { à } 6 \\
\text { mo is }\end{array}$ & $\begin{array}{l}\text { Gènéralisé, absent en surface, } \\
\text { permanent au-dessous de } 80 \mathrm{~cm} \\
\text { Submersion peu prolongée ou nul- } \\
\text { le }:<3 \text { mo is }\end{array}$ & $\begin{array}{l}\text { Généralisè, absent en surface, } \\
\text { permanent au-dessous des drains } \\
\text { Pas de submersion }\end{array}$ \\
\hline $\begin{array}{l}\text { CARACTERISTIQUES AGRONOMIQUES } \\
\text {. Facteur limitant }\end{array}$ & $\begin{array}{l}\text { Très sensibles a l'aspnyxie } \\
\text { Insensides a la sécheresse es- } \\
\text { tivale } \\
\text { Portance tres faible, mécanisa- } \\
\text { tion inossible, drainage réces- } \\
\text { saire }\end{array}$ & $\begin{array}{l}\text { Sensibles à l'asphyxie } \\
\text { Insensibles a la sécheresse es- } \\
\text { tivale } \\
\text { Portance faible, mécanisation } \\
\text { limitee, drainage nécessaire }\end{array}$ & $\begin{array}{l}\text { Peu sensibles a l'asphyxie } \\
\text { Insensibles à la sécheresse es- } \\
\text { tivale } \\
\text { Portance moyenne, mécanisation } \\
\text { possible. drainage pas toujours } \\
\text { nécessaire }\end{array}$ & $\begin{array}{l}\text { Insensibles à l'asphyxie } \\
\text { Insensibles à la secheresse es- } \\
\text { tivale } \\
\text { Portance bonne. mécanisation } \\
\text { possible. gràce au drainage }\end{array}$ \\
\hline
\end{tabular}


Tableau Illb. Typologie simplifiée des sols de versants en Margeride.

\begin{tabular}{|c|c|c|c|c|c|}
\hline 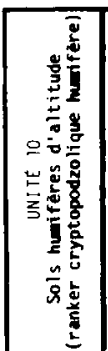 & 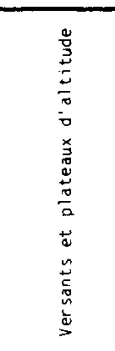 & 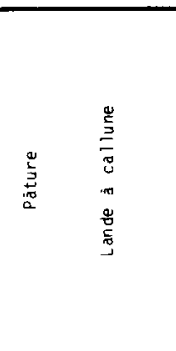 & 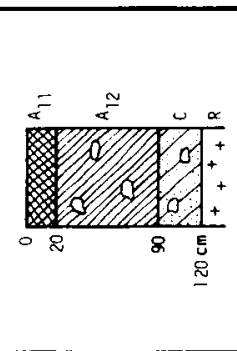 & 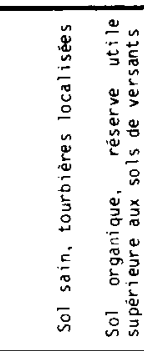 & 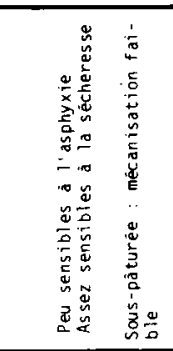 \\
\hline 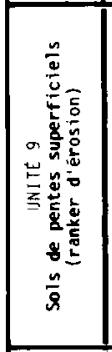 & 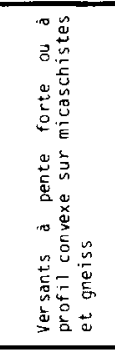 & 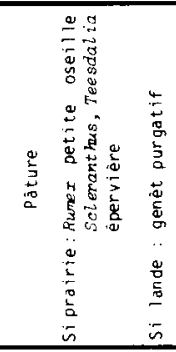 & $\frac{\sqrt{2}}{2}$ & 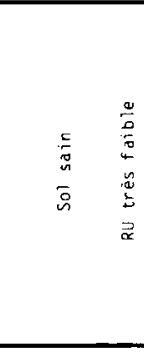 & 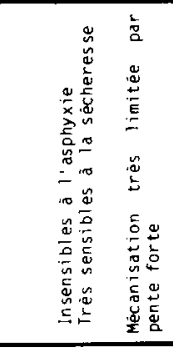 \\
\hline 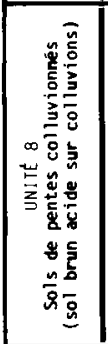 & 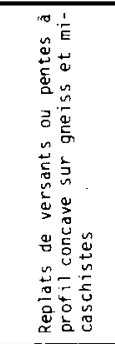 & 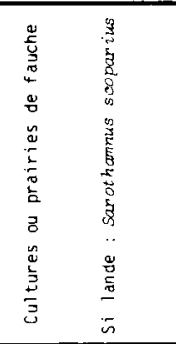 & 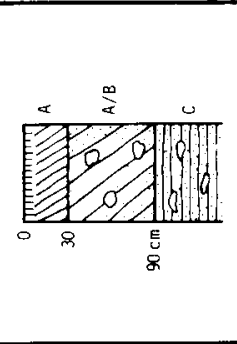 & 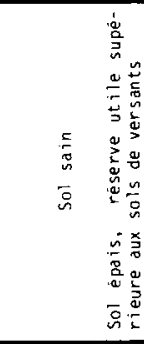 & 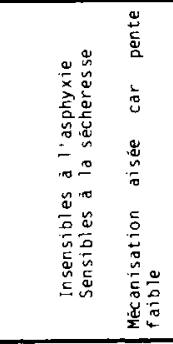 \\
\hline 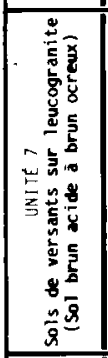 & 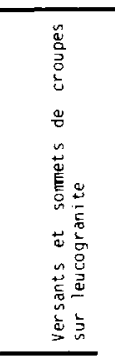 & 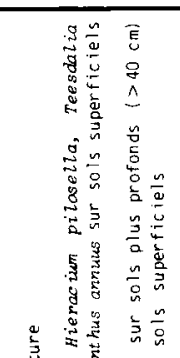 & 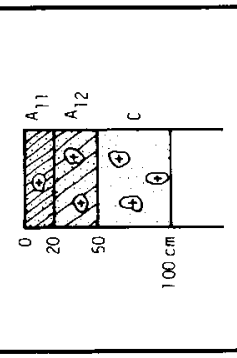 & 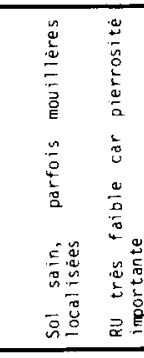 & 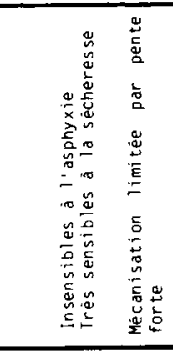 \\
\hline 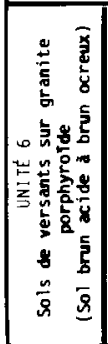 & 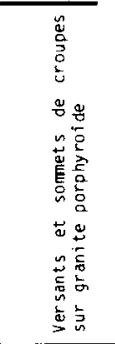 & 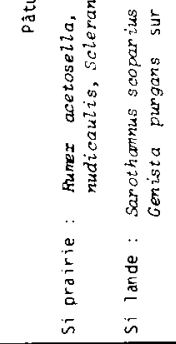 & 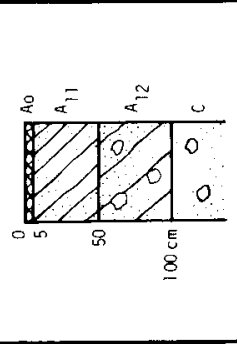 & 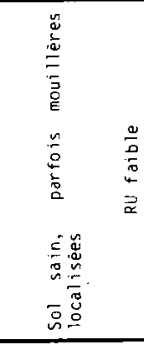 & 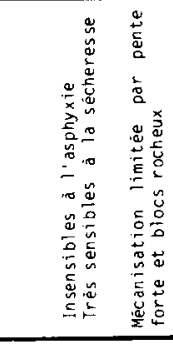 \\
\hline 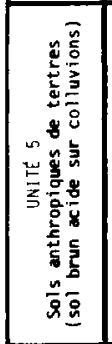 & 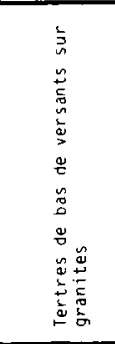 & 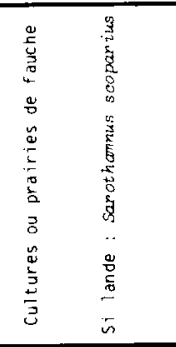 & 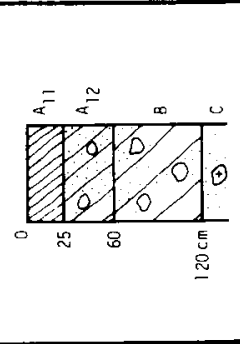 & 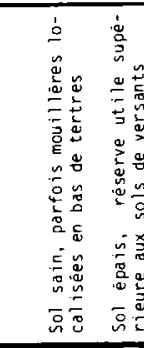 & 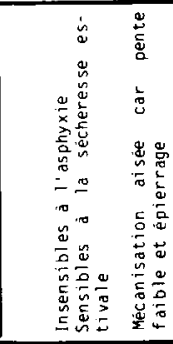 \\
\hline 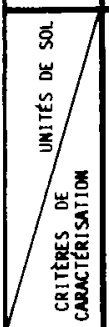 & 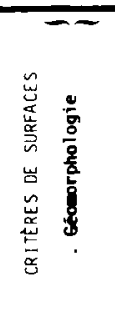 & 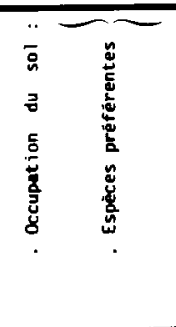 & 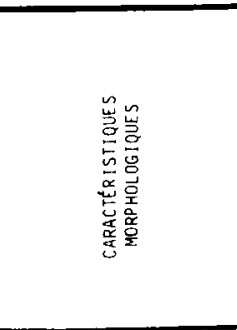 & 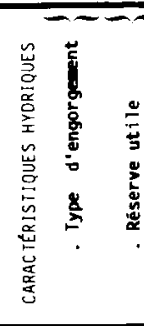 & 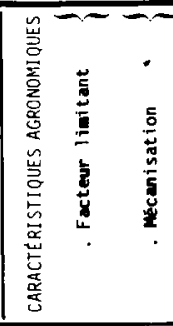 \\
\hline
\end{tabular}


favorisant la création d'une nappe permanente.

\section{Caractéristiques communes}

On a déjà indiqué plus haut les principaux horizons de diagnostics permettant de différencier chacune des diverses unités de sols. De plus, les unités de sols regroupées dans cet ensemble présentent un certain nombre de caractéristiques communes. Tous ces sols présentent un développement des profils dans lesquels alternent des horizons aquifères à texture grossière et des horizons planchers imperméables. La zone d'engorgement d'un gris bleuté (gley caractérisé) est située à plus de $80 \mathrm{~cm}$ de profondeur; elle est surmontée par des niveaux à taches rouilles, plus ou moins proches de la surface, correspondant à la zone de fluctuation de la nappe.

Ces sols présentent une texture fine souvent argilo-limoneuse, avec des horizons de surface de couleur foncée (brun à brun noir), très riches en matières organiques $(15 \%$ en général, $60 \%$ pour les horizons tourbeux). Leur profondeur potentielle est importante $(>1,5 \mathrm{~m})$, mais l'exploitation par les racines est moindre compte tenu de la présence des nappes dans le cas des unités de sols non assainies par drainage.

Le rapport $\mathrm{C} / \mathrm{N}$ reste compris entre 11 et 12,5 dans la zone de fluctuation de la nappe, mais il dépasse 15 dans les horizons tourbeux toujours engorgés.

La capacité d'échange en bases, liée à la teneur en matière organique est élevée (> 2.5 meq/100g). Le complexe absorbant est cependant désaturé $(\mathrm{S} / \mathrm{T}>30 \%)$ avec un $\mathrm{pH}$ qui varie entre 5 et 5,5. Les horizons de surface sont bien pourvus en potassium et en magnésium mais naturellement pauvres en acide phosphorique.

\section{Caractéristiques hydriques}

Ces sols sont soumis à un engorgement généralisé, lié à la présence d'une nappe permanente. Cette nappe, soutenue par la rivière est alimentée par des apports latéraux d'eau issus des versants adjacents (Sattler, 1984). Lorsque aucun système de drainage n'est en place, la longueur des périodes de submersion et la durée de l'engorgement des horizons de surface dépendent de la pente longitudinale de la rivière et de l'efficacité de l'exutoire. Pour chaque unité on a pu définir un régime hydrique assez spécifique qui est en bonne liaison avec les caractéristiques morphologiques des profils des sols observées.

Contraintes et qualités agronomiques majeures Aux caractéristiques que nous venons d'évoquer sont associées un certain nombre de qualités et de facteurs contraignants pour la conduite des prairies. Le premier type de contrainte est l'excès d'eau qui est caractérisé par la présence de plantes hydrophiles, absentes sur les sols de versants et communes aux 4 unités de sols (1 à 4) parmi lesquelles nous avons déterminé :

- des graminées : Houlque laineuse (Holcus lanatus), Vulpin (Alopecurus pratensis), Phléole (Phleum pratense);

- des papilionnacées : Lotier des marais (Lotus ultiginosus);

- des espèces diverses : Bugle rampant (Ajuga reptans), Renouée bistorte (Polygonum bistorta), Scorzonère (Scorzonera humilis), Lychnis fleur de coucou (Lychnis flos-cuculi), Myosotis des marais (Myosotis palustris), Valériane dioïque (Valeriana dioica).

Cet excès d'eau constitue un obstacle majeur à la mécanisation et au travail du sol, car il limite la portance et les durées des périodes d'accès aux parcelles. Cette contrainte est décroissante de l'unité 1 à l'unité 3 , elle devient nulle pour l'unité 4 .

Texture fine, bonne profondeur potentielle exploitable et bonne capacité de réserve hydrique (à la fois intrinsèque et grâce à la présence d'une nappe) rendent ces diverses unités de sols peu sensibles à la sécheresse estivale, de sorte qu'elles ne sont jamais laissées en friche et sont généralement bien exploitées en prés de fauche ou en pâtures.

\section{Les sols de versants}

La différenciation entre les unités de sols de versants est basée sur trois critères : l'altitude, la roche mère (et son modelé associé) et la profondeur (Tableau II). Ainsi, l'on peut facilement distinguer les sols d'altitude (unité $n^{\circ} 10$ ) de l'ensemble des autres sols. Puis, nous avons différencié, en fonction des types de roches, des sols de versants sur granite (Margeride S.S. : unités nos 5, 6, et 7 ) et les sols sur roches métamorphiques (Boraldes: unités nos 8 et 9 ), de texture globalement plus limoneuse et moins graveleuse, de couleur plus foncée, surtout sur les micaschistes (brun noir) et situés sur des pentes plus fortes. Enfin, les sols sur granite porphyroïde (unité 6) se dissocient des sols sur leucogranite (unité 7) par leur faciès global d'altération. Le granite porphyroïde s'altère en boule (de l'ordre du $\mathrm{m}^{3}$ ) et constitue une arène plutôt graveleuse; les sols ont une pierrosité faible, mais contiennent de gros blocs rocheux («les boules"). Par contre, le leucogranite, intrusif et 
moins altérable, formant souvent le haut des buttes, s'altère en éléments plus fins (de l'ordre du $\mathrm{dm}^{3}$ et donne une arène plutôt sableuse. Les sols sur leucogranite sont sensiblement moins profonds, ont une pierrosité plus importante ("carrière») et contiennent aussi moins de gros blocs rocheux que les sols sur granite porphyroïde (Fig. 4).

Par ailleurs, la profondeur du sol nous a aussi amenés à distinguer les sols superficiels (pentes métamorphiques : unité 9 et versants granitiques : unités 6 et 7) des sols profonds et colluvionnés (sur roches métamorphiques : unité 8 et tertres sur granite : unité 5). Le Tableau lllb présente de manière synthétique les caractéristiques des diverses unités de sols, dont nous venons de rap- peler brièvement les critères essentiels de distinction.

\section{Matériau originel et modelé associé}

Les sols regroupés dans cet ensemble sont situés soit sur des pentes faibles pour les zones de bas de versants (tertres), et pour celles du sommet des croupes, soit sur des pentes moyennes à fortes pour les versants granitiques, gneissiques ou micaschisteux.

Cas des granites. Les sols sont développés directement sur le granite sain en place («carrière"), ou le plus souvent sur une arène granitique graveleuse à sableuse («schistras», «tanas»), à structure particulaire, très perméable (Fig. 4).
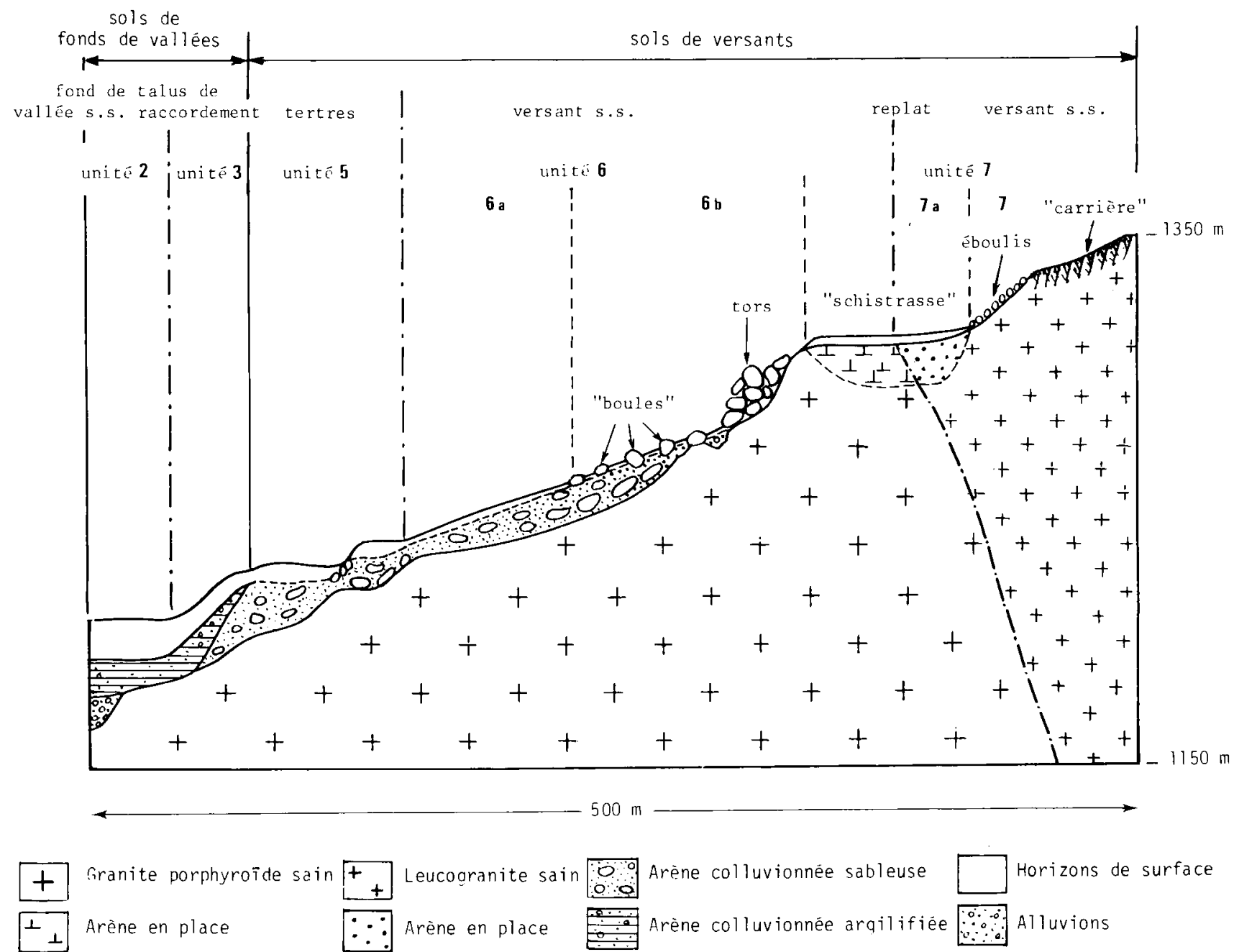

Les numéros correspondent aux unités de sols définies dans la typologie (cf. tableau II)

Fig. 4. Distribution des unités de sols sur un versant granitique (coupe synthétique). 
Cette arène peut être autochtone, constituée par altération in situ du granite, ou bien allochtone, transportée et remaniée le long des versants par des mouvements de solifluxion et surmontant une couche d'arène en place. Dans le premier cas, les particules sableuses sont étroitement imbriquées les unes dans les autres, constituant un horizon très compact, totalement impénétrable par les racines sauf au niveau d'anciennes diaclases. Dans le second cas, l'arène, remaniée et ameublie lors de son transport, acquiert une structure particulaire, plus facilement colonisée par les racines.

Granite sain, arène en place et arène remaniée sont distribués de manière très hétérogène sur les versants avec une logique de distribution dans le paysage que l'on peut schématiser comme suit. Globalement, les sols sur granite non ou peu altéré sont situés sur les pentes fortes et convexes, l'arène d'altération étant décapée par l'érosion. Les sols sur arène en place se trouvent sur les entablements culminaux à pentes faibles. Les sols sur arène remaniée, de loin les plus nombreux, se développent le long de versants à pente faible à moyenne (au niveau des ruptures de pente-tertres successifs) et dans les zones à profil concave.

Cas des gneiss et des micaschistes. Les possibilités d'altération et d'apparition des arènes sont beaucoup plus limitées. On a deux situations extrêmes : d'un côté les pentes très fortes sur gneiss (recreusement des vallées) et les pentes fortes au voisinage du sommet des plateaux (zone de contact gneiss-micaschistes), d'un autre côté, les pentes moyennes des plateaux. Dans les premiers cas, les processus d'érosion dominent largement avec de vastes zones d'affleurements et des sols qui restent très superficiels et localement très caillouteux. Dans les seconds cas, les phénomènes d'altération s'expriment un peu mieux (30 à $40 \mathrm{~cm}$ ) avec apparition de sols plus épais qui dépassent rarement $60 \mathrm{~cm}$, sauf dans certaines zones colluvionnées.

\section{Caractéristiques morphologiques}

Si l'on fait exception des sols de tourbières sur les plateaux d'altitude, les sols de versants possèdent des horizons de surface peu développés et beaucoup moins humifères que les sols des fonds de vallées. Ils sont caractérisés par une couleur brun à brun jaune, un pourcentage assez élevé d'éléments grossiers, en particulier de graviers $(2 \mathrm{~mm}$ à $2 \mathrm{~cm}$ ) qui atteint souvent 10 à $30 \%$ dès la surface. Leur profondeur est très variable en fonction des degrés d'altération, eux-mêmes en relation avec la position occupée le long des versants.

\section{Caractéristiques physico-chimiques}

Exception faite de l'unité 10 , le taux de matière organique est voisin de $5 \%$, voire parfois inférieur même sous prairie. Le $\mathrm{C} / \mathrm{N}$, compris entre 8 et 11 , est l'indice d'une minéralisation de la matière organique plus poussée que dans les sols de fonds de vallée.

La capacité d'échange en bases se situe à des valeurs moyennes voisines ou inférieures à 15 $\mathrm{meq} / 100 \mathrm{~g}$. Le $\mathrm{pH}$ est compris entre 4 et 5,5 avec un complexe absorbant cependant moins désaturé que dans les sols de fonds de vallées. $\mathrm{S} / \mathrm{T}$ reste compris entre 25 et $50 \%$ pour les horizons de surface. La richesse naturelle du sol en $\mathrm{K}$ et $\mathrm{Mg}$ (plus nette sur les granites que sur les micaschistes) (Couturie, 1977) et la fertilisation potassique confèrent aux horizons de surface de bonnes teneurs en $\mathrm{K}$ et $\mathrm{Mg}$. Malgré la pauvreté naturelle du sol en phosphore, les teneurs en $\mathrm{P}_{2} \mathrm{O}_{5}$ sont assez souvent moyennes suite au bon niveau de fertilisation phosphorique que l'on observe surtout sur les versants mécanisables.

\section{Caractéristiques hydriques}

Des "mouillères" localisées existent dans ces positions; mais, de façon générale, les sols de versants sont sains. La réserve utile en eau de ces sols se situe à un niveau moyen ou faible, compte tenu de la texture grossière observée et des teneurs en matière organique, jamais élevées : elle est en liaison directe avec la profondeur des sols.

\section{Caractéristiques agronomiques}

Du fait de l'absence d'engorgement, les prairies ne sont jamais en conditions d'asphyxie. Par contre, elles sont très sensibles à la sécheresse estivale, la réserve utile en eau se situant à des niveaux assez bas. Sur le plan de la flore, plusieurs espèces sont présentes sur l'ensemble des unités $5,67,8$ et 9 , et caractérisent l'absence d'engorgement. On peut citer de façon très schématique :

- des graminées : Dactyle (Dactylis glomerata);

- des espèces diverses : Bugle de Genève (Ajuga genevensis), Armeria (Armeria plantaginea), Helianthème (Helianthernum vulgare), Thym (Thymus serpyllum), CEillet des chartreux (Dianthus carthusianorum).

Dans ces diverses situations des sols de versants, on observe essentiellement des prairies de fauche et des pâtures. Néanmoins, sur les pentes les plus faibles et en bas de versants (tertres) correspondant aux unités 5 et 8 existent des cultures 
localisées. Les obstacles majeurs à une exploitation rationnelle résident dans les difficultés de mécanisation liées à la pente et/ou aux teneurs excessives en blocs ou pierres : les facteurs limitants sont plus ou moins exprimés selon les unités comme mentionné dans le Tableau Illb.

\section{Organisation et répartition des sols dans les pay- sages}

Les diverses unités de sols étant définies et caractérisées, il reste maintenant à préciser comment elles s'organisent et se distribuent au sein des principales unités de paysages.

\section{Cas des vallées}

La zone granitique représente plus de $80 \%$ du territoire de Margeride. Aussi, nous insisterons essentiellement sur ces secteurs. La Figure 5 permet d'illustrer de manière très démonstrative l'existence de 2 grands types de vallées : vallées à fond plat (en auge) et vallées en berceau.

- Les vallées à fond plat ont une largeur de 50 à $500 \mathrm{~m}$. La pente transversale y est faible à nulle; aussi la nappe d'accompagnement de la rivière est toujours proche de la surface. De plus, la pente longitudinale est également très faible, de sorte que la rivière divague en de nombreux méandres et, au printemps, inonde souvent une grande partie de la vallée. Dans ces conditions, les sols hydromorphes dès la surface (unité $n^{\circ} 2$ ) sont nettement dominants. Ce n'est qu'au niveau des talus de raccordement entre vallées et versants qu'existent des portions non inondables situées au-dessus du niveau de fluctuation de la nappe : on y observe des sols à hydromorphie de profondeur (unité 3) et en continuant vers les bas de versants, des sols plus profonds et autrefois cultivés (unité 5) correspondants aux "tertres" colluviaux et anthropiques, qui occupent des superficies non négligeables.

- Les vallées en berceau ont des largeurs (20 à $100 \mathrm{~m}$ ) encore plus réduites que les vallées en auge. La pente transversale est de 3 à $10 \%$; dès que l'on se rapproche des versants, on échappe rapidement à la zone de fluctuation de la nappe de la rivière. De plus, comme la pente longitudinale est souvent accentuée, l'efficacité de l'exutoire est renforcée. Dans ces situations, les sols à hydromorphie de profondeur (unité $n^{\circ} 3$ ) dominent largement alors que les sols plus hydromorphes de l'unité 1 sont localisés au voisinage immédiat des bordures de la rivière et aux zones de confluence entre vallées. Les sols de l'unité 5 sont pratiquement non représentés en raison des pentes fortes et de l'exiguilté des vallées.

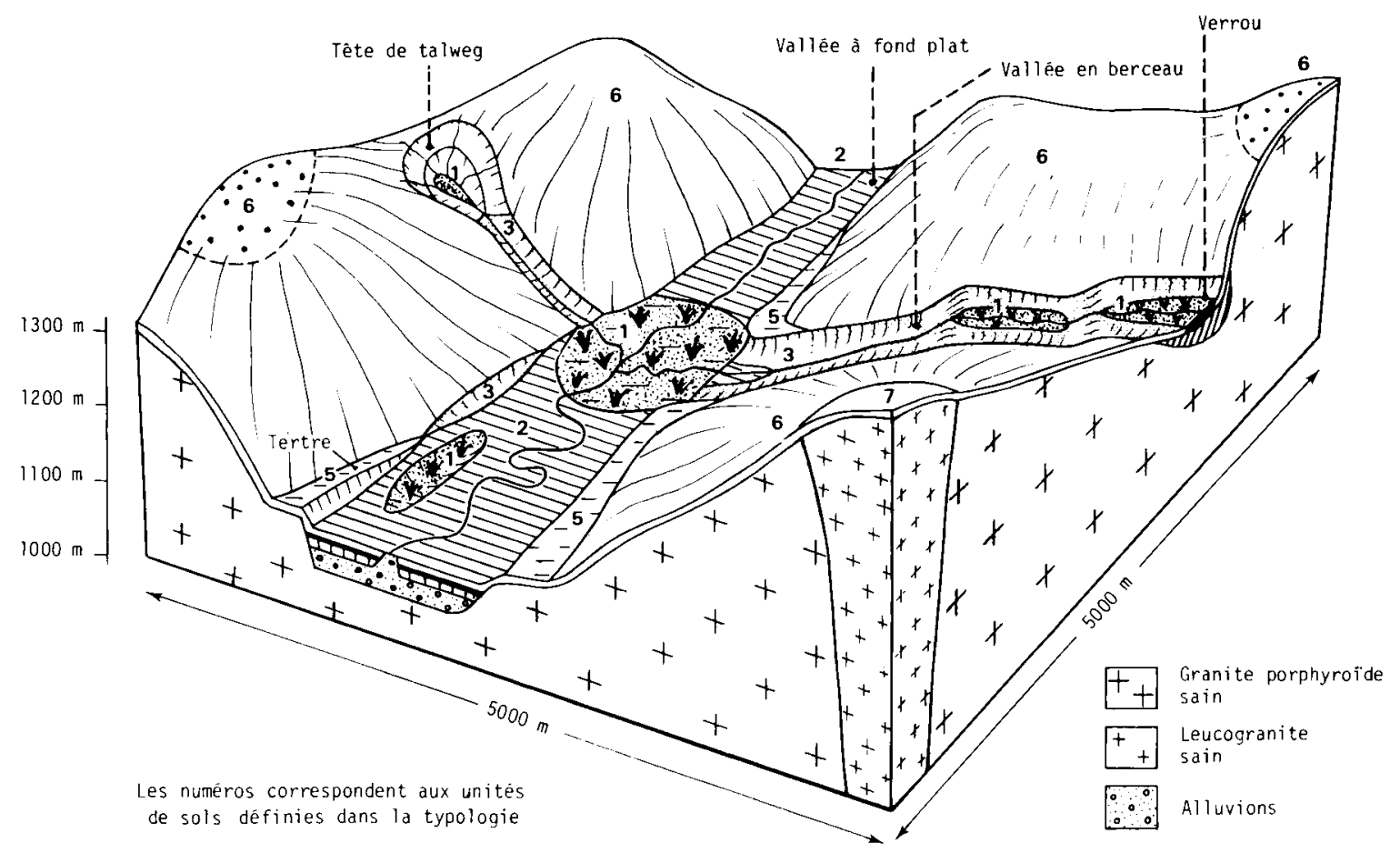

Fig. 5. Les sols dans les paysages : organisation des divers types de vallées en Margeride granitique. 
Les vallées en berceau, avec leur tête de thalweg en cirque, quelquefois reliées à des verroux ou ombilics, se jettent dans les vallées en auge. L'agencement de ces 2 types de vallées représente un ordonnancement assez typique des modelés périglaciaires hérités du dernier épisode glaciaire quaternaire. Sous le climat assez humide de Margeride, les sols tourbescents (unité 1) restent cependant localisés aux zones à faibles pentes longitudinales présentant des possibilités d'évacuation des eaux insuffisantes. Dans les vallées en berceau, les sols tourbescents sont situés daris les systèmes "verrou-ombilic" et dans les têtes de thalwegs. Dans les vallées en auge, ils occupent des superficies plus importantes correspondant aux zones dépressionnaires et aux zones de confluence.

\section{Cas des versants}

La répartition des sols sur les versants granitiques est bien schématisée dans les Figures 3 et 4 . Sur les colluvions épaisses de bas de versants se localisent des sols bruns acides profonds souvent cultivés (unité 5 ) déjà mentionnés plus haut. Sur les pentes moyennes à faibles, de mi-versant, se développent des sols bruns acides à bruns ocreux associés à des rankers superficiels. Grâce aux cartographies détaillées des sites de références, il a été possible de préciser ce diagnostic pédogénétique et on a distingué plusieurs types de sols au sein de ces ensembles en fonction du type de roche sous-jacent (unités 6 et 7), de la profondeur et de la pierrosité ( $6 b$ et $7 b$ ) ou de l'existence de mouillères localisées ( $6 a$ et $7 a$ ). Au-dessus de $1300 \mathrm{~m}$, dans les monts de la Margeride (Fig. 3) apparaissent des sols ocres podzoliques essertiellement localisés à la hêtraie d'altitude, et par suite non mentionnés dans cette typologie des milieux prairiaux. Puis, sur les replats et les croupes de sommets de versant, se développerit le ranker cryptopodzolique sous landes et callunes (unité 10) ainsi que des tourbières (unité 1) localisées en zones dépressionnaires.

Dans les Boraldes métamorphiques existe une répartition un peu différente des sols, schématisée dans la Fig. 2. L'altitude reste globalement plus faible; par suite, seuls des sols bruns acides sur pente faible à moyenne (unité 8) et des rankers d'érosion (unité 9) sur pente forte se succèdent le long des versants. Là encore, les cartographies détaillées réalisées au $1 / 10000^{e}$ sur les secteurs de référence ont permis de distinguer plusieurs unités au sein de ces ensembles pédogénétiques, pour rendre compte des variations des caractéristiques des sols (type de roches, profondeur et pierrosité : sols profonds sur colluvions gneissiques $8 \mathrm{~b}$ ou micaschistes $8 \mathrm{c}$; sols superficiels sur micaschiste 9a).

\section{Extension spatiale régionale des divers types de sols}

Seule une cartographie des sols réalisée à une échelle suffisamment détaillée voisine du 10000 e pourrait permettre de fournir une véritable estimation de l'extension spatiale des divers types de sols inventoriés en Margeride et leur localisation précise. Mais, dans ces zones d'agriculture pauvre, aucun document de ce type n'existe sur des superficies importantes et leur obtention rapide n'est guère prévisible dans la conjoncture agricole actuelle. C'est pourquoi l'on a été amené à proposer la méthode d'approche décrite ici dont l'objectif premier est bien de réaliser une typologie des sols mais qui peut également servir de support à une estimation approchée de la superficie occupée par chacun des types de sols (intérêt statistique).

Une des données fondamentales qui sert de base à cette appréciation spatiale est la cartographie détaillée des sols réalisée sur les secteurs de références. L'on n'a pas pu présenter ici le résultat de ces cartographies pour des raisons évidentes de place disponible. Mais il nous paraît plus important d'insister sur les raisons qui ont motivé le choix des différents secteurs. Ce choix tient compte de la diversité des roches mères présentes et de la diversité des situations altitudinales et géomorphologiques (Tabl. I). Pour chacune des situations représentatives de la Margeride, on dispose donc au moins d'une cartographie détaillée qui fournit des renseignements précieux sur les lois générales de distribution et de répartition des diverses unités de sols précédemment inventoriées.

L'appréciation spatiale des surfaces des diverses unités de sols est donc basée sur l'utilisation conjointe et complémentaire de 3 types de documents :

- carte de reconnaissance des unités de solspaysages au $1 / 250000 \mathrm{e}$;

- cartes géologiques et géomorphologiques au $1 / 50000$ e quand elles existent;

- enfin cartes détaillées des sols des secteurs de références.

L'estimation fournie prend comme hypothèses que les cartographies détaillées sont effectuées dans des secteurs bien représentatifs des unités 
sols-paysages à caractériser et qu'elles ont été effectuées en nombre suffisant. La qualité des extrapolations a été vérifiée dans un certain nombre de situations de Margeride en comparant nos chiffres avec les résultats obtenus par des travaux de cartographie portant sur des surfaces un peu plus importantes (Sattler, 1984) que celles de nos propres travaux. L'approximation obtenue était voisine de $15 \%$. Mais ce chiffre n'a qu'une valeur indicative, car il privilégie beaucoup les milieux alluviaux aux dépens des versants.

Le Tableau IV rassemble les résultats de ces estimations de surfaces couvertes par chaque unité de sols.

\section{Conclusion}

Pour réaliser une typologie des sols des milieux prairiaux, une approche à deux niveaux de perception a été tentée. L'approche à petite échelle (1/250 000e) de la Margeride permet de réaliser un zonage et une première caractérisation des grandes régions naturelles des sols-paysages. Cette étude ne prend toute sa valeur que dans la mesure où des études de sols à grande échelle $\left(1 / 10000^{e}\right)$ sont pratiquées sur chacune de ces grandes régions, au sein de secteurs que l'on peut considérer comme bien représentatifs des situations locales de roches, d'altitude, et de géomorphologie. Ce type d'approche à échelles emboîtées permet d'alléger considérablement la démarche cartographique dans les secteurs où l'on ne dispose pas encore de documents sur les sols, tout en assurant un bon support aux raisonnements scientifiques et notamment agronomiques.

Au terme de l'étude présentée en Margeride, 10 catégories principales de sols ont été décrites. En confrontant nos propres observations avec celles des agriculteurs locaux et des agronomes spécialistes de ces milieux, ces catégories semblent pouvoir se regrouper en 5 grands types de comportement agronomique originaux schématisés ciaprès (voir Tableau V).

Tableau IV. Importance relative des divers types de sols dans la Margeride et les Boraldes.

\begin{tabular}{|c|c|c|}
\hline \multirow[t]{2}{*}{ Nos d'unités de sols } & \multicolumn{2}{|c|}{ Région concernée } \\
\hline & $\begin{array}{l}\text { Margeride } \\
\%\end{array}$ & $\begin{array}{l}\text { Boraldes } \\
\%\end{array}$ \\
\hline $1:$ Sols tourbescents & 5 & - \\
\hline 2 : Sols hydromorphes en surface & 7 & - \\
\hline 3: Sols hydromorphes en profondeur & 7 & - \\
\hline 4: Sols draînés & 1 & - \\
\hline $5:$ Sols de tertres & 5 & - \\
\hline 6 : Sols de versants sur granite porphyroïde & 10 & - \\
\hline $7:$ Sols de versants sur leucogranite & 55 & - \\
\hline 8: Sols de pentes coluvionnés & - & 25 \\
\hline 9 : Sols de pentes superficiels & - & 75 \\
\hline $10:$ Sols humifères d'altitude & 10 & - \\
\hline
\end{tabular}

Tableau V. Les grands types de comportements agronomiques originaux.

\begin{tabular}{|c|c|c|c|}
\hline & \multirow{2}{*}{$\begin{array}{l}\text { Sols de } \\
\text { Margeride }\end{array}$} & \multicolumn{2}{|c|}{ Sensibilité des prairies à } \\
\hline & & l'asphyxie & $\begin{array}{l}\text { la sécheresse } \\
\text { estivale }\end{array}$ \\
\hline $\begin{array}{l}\text { Sols hydromorphes de } \\
\text { fonds de vallées }\end{array}$ & Unités 1, 2, 3 & Sensibles & Non sensibles \\
\hline $\begin{array}{l}\text { Sols drainés de fonds de vallées } \\
\text { Sols sains profonds de versants } \\
\text { Sols sains superficiels } \\
\text { Sols humifères d'altitude }\end{array}$ & $\begin{array}{l}\text { Unité } 4 \\
\text { Unités } 5,8 \\
\text { Unités } 6,7,9 \\
\text { Unité } 10\end{array}$ & $\begin{array}{l}\text { Peu sensibles } \\
\text { Non sensibles } \\
\text { Non sensibles } \\
\text { Peu sensibles }\end{array}$ & $\begin{array}{l}\text { Peu sensibles } \\
\text { Non sensibles } \\
\text { Très sensibles } \\
\text { Assez sensibles }\end{array}$ \\
\hline
\end{tabular}


La typologie des sols mise en place peut d'abord servir de base à une étude statistique à mener en collaboration avec les agronomes sur les productivités prairiales régionales et où l'on testera la pertinence des comportements proposés. Elle peut aussi permettre de complèter un programme d'expérimentations contrôlées sur les problèmes d'implantation et de fertilisation des prairies.

\section{Références}

Bonfils P. \& Legros J.P. (1985) Cartographie de Reconnaissance des Sols de Lozère au 1/250 000. Travaux SESCPF et région programme Languedoc-Roussillon

Couturie J.P. (1977) Le massif granitique de la Margeride. (Thèse de Doctorat) Ann. Sci. Univ. Clermont-Ferrand 62,29 fasc.

Estienne P. (1956) Recherches sur le climat du Massif central français. In : Memorial de la Météorologie Nationale. Thèse, Paris
Fel A. (1962) Les Hautes Terres du Massif Central. PUF, Paris

Fel A. (1983) Personnalité de la Margeride. In : La Margeride : la Montagne et les Hommes. INRA, pp. 21-28

Gachon L. (1946) Les variétés régionales du climat dans le Massif central et le vrai Massif central climatique. Rev. Géogr. Lyon, 33-54

INRA (1983) La Margeride : la Montagne et les Hommes, INRA

Menier D. (1985) Typologie des sols de Margeride. Etude des relations entre facteurs du milieu et comportement prairial. DAA Science du Sol, ENSA, Montpellier

Montard F.X. (de), Daget P. \& Poissonnet J. (1983) Eléments sur les climats, les sols et les types de végétation. In : La Margeride : la Montagne et les Hommes, INRA

RGA (1979-1980-1981) Renseignements Statistiques sur l'Agriculture. Résultats Définitifs. Ministère de l'Agriculture

Sattler P. (1984) Etude Préalable au Drainage des Terres Agricoles. Département de la Lozère. Secteur de Référence de la Margeride. Opération Drainage. Rapport d'étude, Ministère de l'Agriculture 